Pseudo asymptotic behavior of mild solution for semilinearfractional integro-differential equations

\author{
Zhinan Xia
}




\title{
PSEUDO ASYMPTOTIC BEHAVIOR OF MILD SOLUTION FOR SEMILINEAR FRACTIONAL INTEGRO-DIFFERENTIAL EQUATIONS
}

\author{
ZHINAN XIA
}

Received 14 April, 2014

\begin{abstract}
In this paper, by the weighted ergodic function based on the measure theory, we study the pseudo asymptotic behavior of mild solution for semilinear fractional integro-differential equations. The existence, unique of $\mu$-pseudo anti-periodic ( $\mu$-pseudo periodic, $\mu$-pseudo almost periodic, $\mu$-pseudo almost automorphic) solution are investigated. Moreover, an application to fractional partial differential equations is given.
\end{abstract}

2010 Mathematics Subject Classification: 47G05; 35B40

Keywords: semilinear fractional integro-differential equation, pseudo asymptotic, sectorial operator, measure theory

\section{INTRODUCTION}

The study of asymptotic behavior of solutions is one of the most interesting and important topics in the qualitative theory of differential equations. Particularly, pseudo asymptotic behavior of solutions, such as pseudo anti-periodicity; pseudo periodicity; pseudo almost periodicity; pseudo almost automorphy have received much attention in recent years. Many authors have made important contributions to this theory. With the help of weighted measure [10], the notion of weighted class of functions, such as weighted pseudo anti-periodic [2]; weighted pseudo periodic [2]; weighted pseudo almost periodic [10]; weighted pseudo almost automorphic [6] are introduced and applications to differential equations are investigated.

Recently, Blot, Cieutat and Ezzinbi [4,5] use the results of the measure theory to establish $\mu$-ergodic and introduce the new concepts of $\mu$-pseudo almost periodic function, $\mu$-pseudo almost automorphic function, which are more general than weighted pseudo almost automorphic function, weighted pseudo almost automorphic

This research is supported by the National Natural Science Foundation of China (Grant No.11426201) and the Natural Science Foundation of Zhejiang Province (Grant No. LQ13A010015).

(c) 2015 Miskolc University Press 
function, respectively. They developed some results like completeness and composition theorems to investigate evolution equations, partial functional differential equations in Banach space.

In this paper, by the method of measure theory [4,5], we introduce the notions of $\mu$-pseudo anti-periodic function, $\mu$-pseudo periodic function, explore fundamental properties and establish composition theorem. Then we conduct further studies on $\mathcal{P}$ class of functions (i.e., $\mu$-pseudo anti-periodic, $\mu$-pseudo periodic, $\mu$-pseudo almost periodic, $\mu$-pseudo almost automorphic) and its applications in semilinear fractional integro-differential equations in Banach space.

The paper is organized as follows. In Section 2, we recall some fundamental results about the notion of $\mathcal{P}$-class of functions including composition theorem. Sections 3 is devoted to the existence and uniqueness of $\mathcal{P}$ mild solutions to semilinear fractional integro-differential equations. In Section 4, an application to fractional partial differential equations is given.

\section{PReliminaries AND BASIC RESUlts}

Let $(X,\|\cdot\|),(Y,\|\cdot\|)$ be two Banach spaces and $\mathbb{N}, \mathbb{Z}, \mathbb{R}$, and $\mathbb{C}$ stand for the set of natural numbers, integers, real numbers, and complex numbers, respectively. $C(\mathbb{R}, X)$ denotes the set of continuous functions from $\mathbb{R}$ to $X$. $B C(\mathbb{R}, X)$ denotes the Banach space of bounded continuous functions from $\mathbb{R}$ to $X$ with the supremum norm.

\subsection{Sectorial operators and Riemann-Liouville fractional derivative}

Definition 1 ([14]). A closed and densely defined linear operator $A$ is said to be sectorial of type $\widetilde{\omega}$ if there exist $0<\theta<\pi / 2, M>0$, and $\widetilde{\omega} \in \mathbb{R}$ such that its resolvent exists outside the sector

$$
\begin{gathered}
\widetilde{\omega}+S_{\theta}:=\{\widetilde{\omega}+\lambda: \lambda \in \mathbb{C},|\arg (-\lambda)|<\theta\}, \\
\left\|(\lambda I-A)^{-1}\right\| \leq \frac{M}{|\lambda-\widetilde{\omega}|}, \quad \lambda \notin \widetilde{\omega}+S_{\theta} .
\end{gathered}
$$

The sectorial operators are well studied in the literature, we refer to [14] for more details.

Definition 2 ([3]). Let $1<\alpha<2$ be given. Let $A$ be a closed and linear operator with domain $D(A)$ defined on a Banach space $X$. We call $A$ is the generator of a solution operator if there exist $\widetilde{\omega} \in \mathbb{R}$ and a strong continuous function $S_{\alpha}: \mathbb{R}^{+} \rightarrow L(X)$ such that $\left\{\lambda^{\alpha}: \operatorname{Re} \lambda>\widetilde{\omega}\right\} \subset \rho(A)$ and $\lambda^{\alpha-1}\left(\lambda^{\alpha}-A\right)^{-1} x=$ $\int_{0}^{\infty} e^{-\lambda t} S_{\alpha}(t) x d t, \operatorname{Re} \lambda>\widetilde{\omega}, x \in X$. In this case, $S_{\alpha}(t)$ is called the solution operator generated by $A$. 
Note that if $A$ is sectorial of type $\widetilde{\omega}$ with $0<\theta<\pi(1-\alpha / 2)$, then $A$ is the generator of a solution operator given by

$$
S_{\alpha}(t):=\frac{1}{2 \pi i} \int_{\gamma} e^{\lambda t} \lambda^{\alpha-1}\left(\lambda^{\alpha}-A\right)^{-1} d \lambda,
$$

where $\gamma$ is a suitable path lying outside the sector $\widetilde{\omega}+S_{\theta}$ ([8]). Recently, Cuesta ([8]) has proved that if $A$ is a sectorial operator of type $\widetilde{\omega}<0$ for some $0<\theta<\pi(1-\alpha / 2)$ $(1<\alpha<2), M>0$, there exists $C>0$ such that

$$
\left\|S_{\alpha}(t)\right\| \leq \frac{C M}{1+|\widetilde{\omega}| t^{\alpha}}, \quad t \geq 0 .
$$

Note that

$$
\int_{0}^{\infty} \frac{1}{1+|\widetilde{\omega}| t^{\alpha}} d t=\frac{|\widetilde{\omega}|^{-1 / \alpha} \pi}{\alpha \sin (\pi / \alpha)}
$$

for $1<\alpha<2$, therefore $S_{\alpha}(t)$ is integrable on $(0, \infty)$.

Definition 3 ([16]). The fractional order integral of order $\alpha>0$ with the low limit $t_{0}>0$ for a function $f$ is defined as

$$
I^{\alpha} f(t)=\frac{1}{\Gamma(\alpha)} \int_{t_{0}}^{t}(t-s)^{\alpha-1} f(s) d s, \quad t>t_{0}, \alpha>0,
$$

provided the right-hand side is pointwise defined on $\left[t_{0}, \infty\right)$, where $\Gamma$ is the Gamma function.

Definition 4 ([16]). Riemann-Liouville derivative of order $\alpha>0$ with the low limit $t_{0}>0$ for a function $f:\left[t_{0}, \infty\right) \rightarrow \mathbb{R}$ can be written as

$$
D_{t}^{\alpha} f(t)=\frac{1}{\Gamma(n-\alpha)} \frac{d^{n}}{d t^{n}} \int_{t_{0}}^{t}(t-s)^{n-\alpha-1} f(s) d s, \quad t>t_{0}, \quad n-1<\alpha<n .
$$

\section{2. $\mu$-ergodic and functions by measure theory}

$\mathscr{B}$ denotes the Lebesgue $\sigma$-field of $\mathbb{R}, \mathcal{M}$ stands for the set of all positive measure $\mu$ on $\mathscr{B}$ satisfying $\mu(\mathbb{R})=\infty$ and $\mu([a, b])<\infty$ for all $a, b \in \mathbb{R}(a \leq b)$. We formulate the following hypothesis:

$\left(H_{0}\right)$ For all $\tau \in \mathbb{R}$, there exist $\beta>0$ and a bounded interval $I$ such that $\mu(\{a+$ $\tau, a \in A\}) \leq \beta \mu(A)$ if $A \in \mathscr{B}$ satisfies $A \cap I=\varnothing$.

Definition 5. Let $\mu \in \mathcal{M}$. A function $f \in B C(\mathbb{R}, X)$ is said to be $\mu$-ergodic if

$$
\lim _{T \rightarrow+\infty} \frac{1}{\mu([-T, T])} \int_{[-T, T]}\|f(t)\| d \mu(t)=0 .
$$

Denote by $\mathcal{E}(\mathbb{R}, X, \mu)$ the set of such functions.

Lemma 1. Let $\mu \in \mathcal{M}$ and satisfies $\left(H_{0}\right)$, then $\mathcal{E}(\mathbb{R}, X, \mu)$ is translation invariant. 
Definition 6. A function $f \in C(\mathbb{R}, X)$ is said to be anti-periodic if there exists a $\omega \in \mathbb{R} \backslash\{0\}$ with the property that $f(t+\omega)=-f(t)$ for all $t \in \mathbb{R}$. The collection of those functions is denoted by $P_{\omega a p}(\mathbb{R}, X)$.

Definition 7. A function $f \in C(\mathbb{R}, X)$ is said to be periodic if there exists a $\omega \in$ $\mathbb{R} \backslash\{0\}$ with the property that $f(t+\omega)=f(t)$ for all $t \in \mathbb{R}$. The collection of those $\omega$-periodic functions is denoted by $P_{\omega}(\mathbb{R}, X)$.

Definition 8 ([11]). A function $f \in \mathrm{C}(\mathbb{R}, X)$ is said to be almost periodic if for each $\varepsilon>0$, there exists an $l(\varepsilon)>0$, such that every interval $I$ of length $l(\varepsilon)$ contains a number $\tau$ with the property that $\|f(t+\tau)-f(t)\|<\varepsilon$ for all $t \in \mathbb{R}$. Denote by $A P(\mathbb{R}, X)$ the set of such functions.

Definition 9 ([7]). A function $f \in \mathrm{C}(\mathbb{R}, X)$ is said to be almost automorphic if for every sequence of real numbers $\left(s_{n}^{\prime}\right)_{n \in \mathbb{N}}$, there exists a subsequence $\left(s_{n}\right)_{n \in \mathbb{N}}$ such that $g(t):=\lim _{n \rightarrow \infty} f\left(t+s_{n}\right)$ is well defined for each $t \in \mathbb{R}$, and $\lim _{n \rightarrow \infty} g\left(t-s_{n}\right)=f(t)$ for each $t \in \mathbb{R}$. Denote by $A A(\mathbb{R}, X)$ the set of such functions.

Next, we recall the functions by the measure theory.

Definition 10. Let $\mu \in \mathcal{M}$. A function $f \in C(\mathbb{R}, X)$ is said to be $\mu$-pseudo antiperiodic if it can be decomposed as $f=g+\varphi$, where $g \in P_{\omega a p}(\mathbb{R}, X)$ and $\varphi \in$ $\mathcal{E}(\mathbb{R}, X, \mu)$. Denote by $P P_{\omega a p}(\mathbb{R}, X, \mu)$ the collection of such functions.

Definition 11. Let $\mu \in \mathcal{M}$. A function $f \in C(\mathbb{R}, X)$ is said to be a $\mu$-pseudo periodic if it can be decomposed as $f=g+\varphi$, where $g \in P_{\omega}(\mathbb{R}, X)$ and $\varphi \in \mathcal{E}(\mathbb{R}, X, \mu)$. Denote by $P P_{\omega}(\mathbb{R}, X, \mu)$ the collection of such functions.

Definition 12 ([5]). Let $\mu \in \mathcal{M}$. A function $f \in C(\mathbb{R}, X)$ is said to be $\mu$-pseudo almost periodic if it can be decomposed as $f=g+\varphi$, where $g \in A P(\mathbb{R}, X)$ and $\varphi \in \mathcal{E}(\mathbb{R}, X, \mu)$. Denote by $P A P(\mathbb{R}, X, \mu)$ the collection of such functions.

Definition 13 ([4]). Let $\mu \in \mathcal{M}$. A function $f \in C(\mathbb{R}, X)$ is said to be $\mu$-pseudo almost automorphic if it can be decomposed as $f=g+\varphi$, where $g \in A A(\mathbb{R}, X)$ and $\varphi \in \mathscr{E}(\mathbb{R}, X, \mu)$. Denote by $P A A(\mathbb{R}, X, \mu)$ the collection of such functions.

Remark 1. ( $i$ ) If the measure $\mu$ is the Lebesgue measure, then the spaces $P P_{\omega a p}(\mathbb{R}, X, \mu), P P_{\omega}(\mathbb{R}, X, \mu), P A P(\mathbb{R}, X, \mu), P A A(\mathbb{R}, X, \mu)$ are the functions: pseudo anti-periodic $\left(P P_{\omega a p}(\mathbb{R}, X)[17]\right)$, pseudo periodic $\left(P P_{\omega}(\mathbb{R}, X)\right.$ [13]), pseudo almost periodic $(P A P(\mathbb{R}, X)[18])$, pseudo almost automorphic $(P A A(\mathbb{R}, X)[15])$, respectively. One can see $[1,4,5]$ for more details.

(ii) Let $\rho(t)>0$ a.e. on $\mathbb{R}$ for the Lebesgue measure. $\mu$ denotes the positive measure defined by

$$
\mu(A)=\int_{A} \rho(t) d t \quad \text { for } A \in \mathscr{B},
$$


where $d t$ denotes the Lebesgue measure on $\mathbb{R}$, then $P P_{\omega a p}(\mathbb{R}, X, \mu)$, $P P_{\omega}(\mathbb{R}, X, \mu), P A P(\mathbb{R}, X, \mu), P A A(\mathbb{R}, X, \mu)$ are the weighted class of functions: weighted pseudo anti-periodic $\left(W P P_{\omega a p}(\mathbb{R}, X)[2]\right)$, weighted pseudo periodic $\left(W P P_{\omega}(\mathbb{R}, X)[2]\right)$, weighted pseudo almost periodic $(W P A P(\mathbb{R}, X)$ [10]), weighted pseudo almost automorphic $(W P A A(\mathbb{R}, X)[6])$, respectively.

Let

$\mathcal{A}(\mathbb{R}, X)=\left\{P_{\omega a p}(\mathbb{R}, X), P_{\omega}(\mathbb{R}, X), A P(\mathbb{R}, X), A A(\mathbb{R}, X)\right\}$

$\mathcal{P}(\mathbb{R}, X, \mu)=\left\{P P_{\omega a p}(\mathbb{R}, X, \mu), P P_{\omega}(\mathbb{R}, X, \mu), P A P(\mathbb{R}, X, \mu), P A A(\mathbb{R}, X, \mu)\right\}$

It is not difficult to see that $f \in \mathcal{P}(\mathbb{R}, X, \mu)$ if and only if it can be decomposed as $f=g+\varphi$, where $g \in \mathcal{A}(\mathbb{R}, X)$ and $\varphi \in \mathcal{E}(\mathbb{R}, X, \mu)$.

Definition 14. Let $\mu_{1}, \mu_{2} \in \mathcal{M}, \mu_{1}$ is said to be equivalent to $\mu_{2}\left(\mu_{1} \sim \mu_{2}\right)$ if there exist constants $\alpha, \beta>0$ and a bounded interval $I$ (eventually $I=\varnothing$ ) such that $\alpha \mu_{1}(A) \leq \mu_{2}(A) \leq \beta \mu_{1}(A)$ for $A \in \mathscr{B}$ satisfies $A \cap I=\varnothing$.

Similarly as the proof of $[4,5]$, we have the following results for the class of functions $\mathcal{P}(\mathbb{R}, X, \mu)$.

Lemma 2. Let $\mu \in \mathcal{M}$, then the following properties hold:

(i) $f \pm g \in \mathcal{P}(\mathbb{R}, X, \mu)$ if $f, g \in \mathcal{P}(\mathbb{R}, X, \mu)$.

(i i) $\lambda f \in \mathcal{P}(\mathbb{R}, X, \mu)$ if $\lambda \in \mathbb{R}, f \in \mathcal{P}(\mathbb{R}, X, \mu)$.

(iii) $\mathcal{P}(\mathbb{R}, X, \mu)$ is a Banach space with the supremum norm $\|\cdot\|$.

$P P_{\omega a p}(\mathbb{R}, X, \mu) \subset P P_{\omega}(\mathbb{R}, X, \mu) \subset P A P(\mathbb{R}, X, \mu) \subset P A A(\mathbb{R}, X, \mu) \subset B C(\mathbb{R}, X)$

Lemma 3. Let $\mu_{1}, \mu_{1} \in \mathcal{M}$. If $\mu_{1} \sim \mu_{2}$, then

$$
\mathcal{E}\left(\mathbb{R}, X, \mu_{1}\right)=\mathcal{E}\left(\mathbb{R}, X, \mu_{2}\right), \quad \mathcal{P}\left(\mathbb{R}, X, \mu_{1}\right)=\mathcal{P}\left(\mathbb{R}, X, \mu_{2}\right) .
$$

Theorem 1. Let $\mu \in \mathcal{M}, f \in \mathcal{P}(\mathbb{R} \times X \times X, X, \mu)$, and satisfies

(i) $f$ is uniformly continuous on each compact set $K_{1} \times K_{2}$ in $X \times X$ with respect to the second, third variable $u, v$.

(ii) For all bounded subset $E_{1}, E_{2}$ of $X, f$ is bounded on $\mathbb{R} \times E_{1} \times E_{2}$. then $f\left(\cdot, h_{1}(\cdot), h_{2}(\cdot)\right) \in \mathcal{P}(\mathbb{R}, X, \mu)$ if $h_{1}(\cdot), h_{2}(\cdot) \in \mathcal{P}(\mathbb{R}, X, \mu)$.

Corollary 1. Let $\mu \in \mathcal{M}, f \in \mathcal{P}(\mathbb{R} \times X \times X, X, \mu)$, and there exists a constant $L_{f}>0$ such that

$$
\begin{gathered}
\left\|f\left(t, u_{1}, u_{2}\right)-f\left(t, v_{1}, v_{2}\right)\right\| \leq L_{f}\left(\left\|u_{1}-v_{1}\right\|+\left\|u_{2}-v_{2}\right\|\right), \\
t \in \mathbb{R}, u_{1}, u_{2}, v_{1}, v_{2} \in X,
\end{gathered}
$$

then $f\left(\cdot, h_{1}(\cdot), h_{2}(\cdot)\right) \in \mathcal{P}(\mathbb{R}, X, \mu)$ if $h_{1}(\cdot), h_{2}(\cdot) \in \mathcal{P}(\mathbb{R}, X, \mu)$. 
Lemma 4. Let $\{S(t)\}_{t \geq 0} \subset L(X)$ be a strongly continuous family of bounded and linear operators such that $\|S(t)\| \leq \phi(t), t \in \mathbb{R}^{+}$, where $\phi \in L^{1}\left(\mathbb{R}^{+}\right)$. If $f \in$ $\mathcal{P}(\mathbb{R}, X, \mu), \mu \in \mathcal{M}$ and satisfies $\left(H_{0}\right)$, then

$$
(\Lambda f)(t):=\int_{-\infty}^{t} S(t-s) f(s) d s \in \mathcal{P}(\mathbb{R}, X, \mu) .
$$

Proof. Let $f(t)=f_{1}(t)+f_{2}(t)$, where $f_{1} \in \mathcal{A}(\mathbb{R}, X), f_{2} \in \mathcal{E}(\mathbb{R}, X, \mu)$, then $(\Lambda f)(t)=\int_{-\infty}^{t} S(t-s) f_{1}(s) d s+\int_{-\infty}^{t} S(t-s) f_{2}(s) d s:=\left(\Lambda_{1} f\right)(t)+\left(\Lambda_{2} f\right)(t)$

where

$$
\left(\Lambda_{1} f\right)(t)=\int_{-\infty}^{t} S(t-s) f_{1}(s) d s, \quad\left(\Lambda_{2} f\right)(t)=\int_{-\infty}^{t} S(t-s) f_{2}(s) d s, \quad t \in \mathbb{R} .
$$

By $f_{1} \in \mathcal{A}(\mathbb{R}, X)$, it is not difficult to see that $\Lambda_{1} f \in \mathcal{A}(\mathbb{R}, X)$. Next, we show that $\Lambda_{2} f \in \mathcal{E}(\mathbb{R}, X, \mu)$, that is,

$$
\lim _{T \rightarrow+\infty} \frac{1}{\mu([-T, T])} \int_{[-T, T]}\left\|\left(\Lambda_{2} f\right)(t)\right\| d \mu(t)=0 .
$$

In fact, for $T>0$, by using Fubini's theorem, one has

$$
\begin{aligned}
& \frac{1}{\mu([-T, T])} \int_{[-T, T]}\left\|\left(\Lambda_{2} f\right)(t)\right\| d \mu(t) \\
& =\frac{1}{\mu([-T, T])} \int_{[-T, T]}\left\|\int_{-\infty}^{t} S(t-s) f_{2}(s) d s\right\| d \mu(t) \\
& \leq \frac{1}{\mu([-T, T])} \int_{[-T, T]} \int_{-\infty}^{t}\left\|\phi(t-s) f_{2}(s)\right\| d s d \mu(t) \\
& =\frac{1}{\mu([-T, T])} \int_{[-T, T]} \int_{0}^{\infty}\left\|\phi(s) f_{2}(t-s)\right\| d s d \mu(t) \\
& \leq \int_{0}^{\infty} \phi(s) \Phi_{T}(s) d s,
\end{aligned}
$$

where

$$
\Phi_{T}(s)=\frac{1}{\mu([-T, T])} \int_{[-T, T]}\left\|f_{2}(t-s)\right\| d \mu(t) .
$$

Since $\mu \in \mathcal{M}$ and satisfies $\left(H_{0}\right)$, by Lemma 1 , it follows that $f_{2}(\cdot-s) \in \mathcal{E}(\mathbb{R}, X, \mu)$ for each $s \in \mathbb{R}$, hence $\lim _{T \rightarrow \infty} \Phi_{T}(s)=0$ for all $s \in \mathbb{R}$. Since $\left\|\Phi_{T}\right\|<\infty$ and $\phi \in$ $L^{1}\left(\mathbb{R}^{+}\right)$, then $\Lambda_{2} f \in \mathcal{E}(\mathbb{R}, X, \mu)$ by using the Lebesgue dominated convergence theorem, so $\Lambda f \in \mathcal{P}(\mathbb{R}, X, \mu)$. 


\section{FRACTIONAL INTEGRO-DIFFERENTIAL EQUATIONS}

This section is devoted to the existence, uniqueness of $\mathcal{P}(\mathbb{R}, X, \mu)$ solution to the following fractional integro-differential equations

$$
\begin{aligned}
& D_{t}^{\alpha} u(t)={ }_{t} A u(t)+D_{t}^{\alpha-1} f(t, u(t), K u(t)), \quad t \in \mathbb{R}, 1<\alpha<2, \\
& K u(t)=\int_{-\infty}^{t} k(t-s) g(s, u(s)) d s .
\end{aligned}
$$

where $A: D(A) \subset X \rightarrow X$ is a linear densely operator of sectorial type on a complex Banach space $(X,\|\cdot\|), f, g, k$ are continuous functions. The fractional derivative $D_{t}^{\alpha}$ is to be understood in Riemann-Liouville sense.

Definition 15 ( $[9,12])$. Assume that $A$ generates an integral solution operator $S_{\alpha}(t)$. A continuous function $u: \mathbb{R} \rightarrow X$ satisfying the integral equation

$$
u(t)=\int_{-\infty}^{t} S_{\alpha}(t-s) f(s, u(s), K u(s)) d s, \quad t \in \mathbb{R},
$$

is called a mild solution on $\mathbb{R}$ to (3.1).

To study (3.1), we introduce the following assumptions:

$\left(H_{1}\right) A$ is a sectorial operator of type $\widetilde{\omega}<0$ with $0<\theta<\pi(1-\alpha / 2)$.

$\left(H_{2}\right) k(t) \in L^{1}\left(\mathbb{R}^{+}\right)$.

$\left(H_{3}\right) g \in \mathcal{P}(\mathbb{R} \times X, X, \mu)$, and there exists a constant $L_{g}>0$ such that

$$
\|g(t, u)-g(t, v)\| \leq L_{g}\|u-v\|, \quad t \in \mathbb{R}, \quad u, v \in X .
$$

( $\left.H_{4}\right) f \in \mathcal{P}(\mathbb{R} \times X \times X, X, \mu)$, and there exists a constant $L_{f}>0$ such that

$$
\begin{gathered}
\left\|f\left(t, u_{1}, u_{2}\right)-f\left(t, v_{1}, v_{2}\right)\right\| \leq L_{f}\left(\left\|u_{1}-v_{1}\right\|+\left\|u_{2}-v_{2}\right\|\right), \\
t \in \mathbb{R}, u_{1}, u_{2}, v_{1}, v_{2} \in X .
\end{gathered}
$$

( $\left.H_{5}\right) \mu \in \mathcal{M}$ and satisfies $\left(H_{0}\right)$.

Lemma 5. If $u \in \mathcal{P}(\mathbb{R}, X, \mu)$, and assume that $\left(H_{1}\right),\left(H_{2}\right),\left(H_{3}\right)$ and $\left(H_{5}\right)$ hold, then $K u \in \mathcal{P}(\mathbb{R}, X, \mu)$.

Proof. Since $g \in \mathcal{P}(\mathbb{R} \times X, X, \mu)$, by $\left(H_{3}\right)$ and Theorem 1 , it is clear that $g(\cdot, u(\cdot)) \in$ $\mathcal{P}(\mathbb{R}, X, \mu)$. Similarly as the proof of Lemma $4, K u \in \mathcal{P}(\mathbb{R}, X, \mu)$.

Theorem 2. Assume that $\left(H_{1}\right)-\left(H_{5}\right)$ hold, if

$$
C M L_{f}\left(1+L_{g}\|k\|_{L^{1}}\right)|\widetilde{\omega}|^{-1 / \alpha} \pi<\alpha \sin (\pi / \alpha),
$$

then (3.1) has a unique mild solution $u(t) \in \mathcal{P}(\mathbb{R}, X, \mu)$. 
Proof. Define the operator $\mathcal{F}: \mathcal{P}(\mathbb{R}, X, \mu) \rightarrow \mathcal{P}(\mathbb{R}, X, \mu)$ by

$$
(\mathscr{F} u)(t)=\int_{-\infty}^{t} S_{\alpha}(t-s) f(s, u(s), K u(s)) d s, t \in \mathbb{R} .
$$

First, we show that $\mathcal{F}$ is well defined. In fact, if $u \in \mathcal{P}(\mathbb{R}, X, \mu)$, by Lemma 5, $K u \in \mathcal{P}(\mathbb{R}, X, \mu)$, then $f(\cdot, u(\cdot), K u(\cdot)) \in \mathcal{P}(\mathbb{R}, X, \mu)$ by Corollary 1 . On the other hand, by (2.1), we have

$$
\left\|S_{\alpha}(t)\right\| \leq \frac{C M}{1+|\widetilde{\omega}| t^{\alpha}}, \quad t \geq 0
$$

Since $1<\alpha<2$ and $C M /\left(1+|\widetilde{\omega}| t^{\alpha}\right) \in L^{1}\left(\mathbb{R}^{+}\right), \mathscr{F}$ maps $\mathcal{P}(\mathbb{R}, X, \mu)$ into itself by Lemma 4.

For any $u, v \in \mathcal{P}(\mathbb{R}, X, \mu)$,

$$
\begin{aligned}
& \|(\mathcal{F} u)(t)-(\mathcal{F} v)(t)\| \\
& \leq \int_{-\infty}^{t}\left\|S_{\alpha}(t-s)\right\|\|f(s, u(s), K u(s))-f(s, v(s), K v(s))\| d s \\
& \leq L_{f} \int_{-\infty}^{t}\left\|S_{\alpha}(t-s)\right\|(\|u(s)-v(s)\|+\|K u(s)-K v(s)\|) d s \\
& \leq L_{f}\left(1+L_{g}\|k\|_{L^{1}}\right) \int_{-\infty}^{t}\left\|S_{\alpha}(t-s)\right\|\|u(s)-v(s)\| d s \\
& \leq L_{f}\left(1+L_{g}\|k\|_{L^{1}}\right)\|u-v\| \int_{0}^{\infty} \frac{C M}{1+|\widetilde{\omega}| s^{\alpha}} d s \\
& \leq \frac{C M L_{f}\left(1+L_{g}\|k\|_{L^{1}}\right)|\widetilde{\omega}|^{-1 / \alpha} \pi}{\alpha \sin (\pi / \alpha)}\|u-v\| .
\end{aligned}
$$

By the Banach contraction mapping principle, $\mathscr{F}$ has a unique fixed point in $\mathcal{P}(\mathbb{R}, X, \mu)$, which is the unique $\mathcal{P}(\mathbb{R}, X, \mu)$ mild solution to (3.1).

Next, we formulate the following hypotheses:

( $\left.H_{6}\right) \quad f \in \mathcal{P}(\mathbb{R} \times X \times X, X, \mu)$ and satisfies

$$
\begin{gathered}
\left\|f\left(t, u_{1}, u_{2}\right)-f\left(t, v_{1}, v_{2}\right)\right\| \leq L_{f}(t)\left(\left\|u_{1}-v_{1}\right\|+\left\|u_{2}-v_{2}\right\|\right), \\
t \in \mathbb{R}, u_{1}, u_{2}, v_{1}, v_{2} \in X .
\end{gathered}
$$

where $L_{f} \in L^{1}\left(\mathbb{R}, \mathbb{R}^{+}\right)$.

$\left(H_{7}\right)$ For all bounded sets $E_{1}, E_{2}$ of $X, f$ is bounded on $\mathbb{R} \times E_{1} \times E_{2}$.

Theorem 3. Assume that $\left(H_{1}\right)-\left(H_{3}\right),\left(H_{5}\right)-\left(H_{7}\right)$ hold, then (3.1) has a unique mild solution $u(t) \in \mathcal{P}(\mathbb{R}, X, \mu)$.

Proof. Define the operator $\mathcal{F}$ as in (3.2). Similarly as the proof Theorem 2, $\mathscr{F}$ is well defined. Lemma 4, Lemma 5 and Theorem 1 imply that $\mathscr{F}$ maps $\mathcal{P}(\mathbb{R}, X, \mu)$ into itself. 
Let $u, v \in \mathcal{P}(\mathbb{R}, X, \mu))$ and define $C_{\alpha}=\sup _{t \in \mathbb{R}^{+}}\left\|S_{\alpha}(t)\right\|$, one has

$$
\begin{aligned}
& \|(\mathcal{F} u)(t)-(\mathcal{F} v)(t)\| \\
& \leq \int_{-\infty}^{t}\left\|S_{\alpha}(t-s)\right\|\|f(s, u(s), K u(s))-f(s, v(s), K v(s))\| d s \\
& \leq \int_{-\infty}^{t} L_{f}(s)\left\|S_{\alpha}(t-s)\right\|(\|u(s)-v(s)\|+\|K u(s)-K v(s)\|) d s \\
& \leq\left(1+L_{g}\|k\|_{L^{1}}\right) \int_{-\infty}^{t} L_{f}(s)\left\|S_{\alpha}(t-s)\right\|\|u(s)-v(s)\| d s \\
& \leq C_{\alpha}\left(1+L_{g}\|k\|_{L^{1}}\right)\|u-v\| \int_{-\infty}^{t} L_{f}(s) d s .
\end{aligned}
$$

Similarly,

$$
\begin{aligned}
& \left\|\mathcal{F}^{2} u-\mathcal{F}^{2} v\right\| \\
& \leq C_{\alpha}\left(1+L_{g}\|k\|_{L^{1}}\right) \int_{-\infty}^{t} L_{f}(s)\|(\mathcal{F} u)(s)-(\mathcal{F} v)(s)\| d s \\
& \leq\left(C_{\alpha}\left(1+L_{g}\|k\|_{L^{1}}\right)\right)^{2}\|u-v\| \int_{-\infty}^{t} L_{f}(s) \int_{-\infty}^{s} L_{f}(\sigma) \mathrm{d} \sigma d s \\
& =\left(C_{\alpha}\left(1+L_{g}\|k\|_{L^{1}}\right)\right)^{2}\|u-v\| \int_{-\infty}^{t}\left(\int_{-\infty}^{s} L_{f}(\sigma) d \sigma\right) d\left(\int_{-\infty}^{s} L_{f}(\sigma) d \sigma\right) \\
& \leq \frac{\left(C_{\alpha}\left(1+L_{g}\|k\|_{L^{1}}\right)\right)^{2}}{2 !}\|u-v\|\left(\int_{-\infty}^{t} L_{f}(s) d s\right)^{2} .
\end{aligned}
$$

By the method of mathematical induction, one has

$$
\left\|\mathscr{F}^{n} u-\mathscr{F}^{n} v\right\| \leq \frac{\left(C_{\alpha}\left(1+L_{g}\|k\|_{L^{1}}\right)\right)^{n}}{n !}\|u-v\|\left(\int_{-\infty}^{t} L_{f}(s) d s\right)^{n} .
$$

Moreover, since $L_{f} \in L^{1}\left(\mathbb{R}, \mathbb{R}^{+}\right)$,

$$
\left\|\widetilde{F}^{n} u-\widetilde{F}^{n} v\right\| \leq \frac{\left(C_{\alpha}\left(1+L_{g}\|k\|_{L^{1}}\right)\left\|L_{f}\right\|_{L^{1}}\right)^{n}}{n !}\|u-v\| .
$$

For sufficiently large $n$, we have $\left(C_{\alpha}\left(1+L_{g}\|k\|_{L^{1}}\right)\|L\|_{L^{1}}\right)^{n} / n !<1$, by the Banach contraction mapping principle, $\mathscr{F}$ has a unique fixed point in $\mathcal{P}(\mathbb{R}, X, \mu)$, which is the unique $\mathcal{P}(\mathbb{R}, X, \mu)$ mild solution to (3.1). The proof is complete. 


\section{EXAMPLE}

Consider the fractional partial differential equation

$$
\begin{aligned}
& D_{t}^{\alpha} w(t, x)=\frac{\partial^{2} w(t, x)}{\partial x^{2}}-a w(t, x)+D_{t}^{\alpha-1} f(t, x, w(t, x), K w(t, x)), \\
& \quad t \in \mathbb{R}, x \in(0,1) \\
& K w(t, x)=\int_{-\infty}^{t} k(t-s) g(s, x, w(s, x)) d s \\
& w(t, 0)=w(t, 1)=0,
\end{aligned}
$$

where $1<\alpha<2, a>0$ and $k(\cdot)$ is a real valued continuous nonincreasing function such that $|k(t)| \leq C_{k} e^{-b t}$ for $t \geq 0$ and $C_{k}, b$ are positive constants.

Let $X=L^{2}[0,1]$ and define the operator $A$ by

$$
\begin{aligned}
& D(A):=\left\{w \in L^{2}[0,1]: w^{\prime \prime} \in L^{2}[0,1], w(0)=w(1)=0\right\}, \\
& A w=w^{\prime \prime}-a w, \quad \text { for all } w \in D(A) .
\end{aligned}
$$

It is well know that $A$ is the infinitisimal generator of an analytic semigroup on $L^{2}[0,1]$ ([14]), thus $A$ is of sectorial type $\widetilde{\omega}=-a<0$.

Let $u(t)=w(t, x), t \in \mathbb{R}, x \in[0,1]$, then (4.1) can be rewritten as the abstract form (3.1). By Theorem 2,

Theorem 4. Under assumptions $\left(H_{3}\right),\left(H_{4}\right)$ and $\left(H_{5}\right)$, then (4.1) has a unique $\mathcal{P}(\mathbb{R}, X, \mu)$ mild solution if $C L_{f}\left(1+L_{g}\|k\|_{L^{1}}\right)|a|^{-1 / \alpha} \pi<\alpha \sin (\pi / \alpha)$.

\section{REFERENCES}

[1] M. Adimy, K. Ezzinbi, and C. Marquet, "Ergodic and weighted pseudo-almost periodic solutions for partial functional differential equations in fading memory spaces," J. Appl. Math. Comput., vol. 44, no. 1-2, pp. 147-165, 2014.

[2] N. S. Al-lslam, S. M. Alsulami, and T. Diagana, "Existence of weighted pseudo anti-periodic solutions to some non-autonomous differential equations," Appl. Math. Comput., vol. 218, no. 11, pp. 6536-6548, 2012.

[3] E. Bazhlekova, Fractional evolution equation in Banach spaces, ser. Ph.D. Thesis. Eindhoven University of Techology, 2001.

[4] J. Blot, P. Cieutat, and K. Ezzinbi, "Measure theory and pseudo almost automorphic functions: New developments and applications," Nonlinear Anal., vol. 75, no. 4, pp. 2426-2447, 2012.

[5] J. Blot, P. Cieutat, and K. Ezzinbi, "New approach for weighted pseudo-almost periodic functions under the light of measure theory, basic results and applications," Appl. Anal., vol. 92, no. 3, pp. 493-526, 2013.

[6] J. Blot, G. M. Mophou, G. M. N'Guérékata, and D. Pennequin, "Weighted pseudo almost automorphic functions and applications to abstract differential equations," Nonlinear Anal., vol. 71, no. 3-4, pp. 903-909, 2009.

[7] S. Bochner, "A new approach to almost periodicity," Proc. Natl. Acad. Sci. USA, vol. 48, no. 12, pp. 2039-2043, 1962.

[8] E. Cuesta, "Asymptotic behaviour of the solutions fractional integro-differential equations and some time discretizations," Discrete Contin. Dyn. Syst., vol. Supplement, pp. 277-285, 2007.

[9] C. Cuevas and C. Lizama, "Almost automorphic solutions to a class of semilinear fractional differential equations," Appl. Math. Lett., vol. 21, no. 12, pp. 1315-1319, 2008. 
[10] T. Diagana, "Weighted pseudo almost periodic functions and applications," C. R. Acad. Sci. Paris, Ser. I, vol. 343, no. 10, pp. 643-646, 2006.

[11] A. M. Fink, Almost Periodic Differential Equations. New York: Springer, 1974.

[12] V. Kavitha, P. Z. Wang, and R. Murugesu, "Existence of weighted pseudo almost automorphic mild solutions to fractional integro-differential equations," Journal of Fractional Calculus and Applications, vol. 4, no. 1, pp. 37-55, 2013.

[13] C. Lizama and G. M. N'Guérékata, "Bounded mild solutions for semilinear integro differential equations in Banach spaces," Integral Equations Operator Theory, vol. 68, no. 2, pp. 207-227, 2010.

[14] A. Lunardi, Analytic Semigroups and Optimal Regularity in Parabolic Problems, ser. Progress in Nonlinear Differential Equations and their Applications. Basel: Birkhäuser, 1995, vol. 16.

[15] G. M. N'Guérékata, Topics in Almost Automorphy. New York: Springer-Verlag, 2005.

[16] I. Podlubny, Fractional Differential Equations. New York: Academic Press, 1999.

[17] Z. N. Xia, "Weighted Stepanov-like pseudoperiodicity and applications," Abstr. Appl. Anal., vol. 2014, pp. 1-14, 2014.

[18] C. Zhang, Pseudo almost periodic functions and their applications, ser. thesis. the University of Western Ontario, 1992.

Author's address

Zhinan Xia

Zhejiang University of Technology, Department of Applied Mathematics, Hangzhou, Zhejiang, 310023, China

E-mail address: xiazn299@zjut.edu.cn 